\title{
Potencial inseticida de óleos de origem vegetal sobre Grapholita molesta (Busck) (Lepidoptera: Tortricidae)
}

COLPO, J.F. ${ }^{* *}$; JAHNKE, S.M. ${ }^{1 *} \&$ FÜLLER, T. ${ }^{1}$.

${ }^{1}$ Universidade Federal do Rio Grande do Sul, Faculdade de Agronomia / Campus Agronomia, Av. Bento Gonçalves, 7712, CEP: 91540-000 Porto Alegre, Brasil.

\begin{abstract}
RESUMO: Em decorrência do estudo das interações químicas entre insetos e herbívoros, e do avanço da pesquisa fitoquímica, é conhecida ampla variedade de produtos naturais com potencial inseticida. Grapholita molesta (Busck) (Lepidoptera: Tortricidae), mariposa-oriental, é uma das principais pragas do pessegueiro, danificando brotações e frutos. O objetivo do presente estudo foi verificar a mortalidade, atratividade e deterrência de óleos vegetais em G. molesta. Ovos e pupas de insetos provindos de criação artificial foram imersos nos óleos essenciais de Elionurus muticus (Spreng.) Kuntze e Cymbopogon winterianus Jowitt ex Bor nas concentrações de $0,25 \%, 0,5 \%, 1 \%$ e $0,5 \%, 1 \%, 5 \%$. Os testes de atratividade foram realizados em olfatômetro do tipo "Y". A deterrência à oviposição foi testada em gaiolas sem escolha e de dupla escolha. O óleo de E. muticus aplicado em ovos de G. molesta nas três concentrações causou mortalidades maiores que $30 \%$, diferindo significativamente dos controles $(p \leq 0,05)$. A mortalidade causada pelo óleo de citronela foi em torno de $70 \%$, significativamente superior ao controle $(p \leq 0,01)$. O óleo de citronela aplicado a $1 \%$ em pupas causou mortalidade de $99,8 \%$. No teste com o olfatômetro, não houve atratividade dos adultos para nenhum dos óleos. $\mathrm{A}$ média de ovos no substrato com o tratamento $(0,33 \pm 0,33)$, foi significativamente menor que no controle $(7,3 \pm 0,88)(p \leq 0,01)$, indicando deterrência à oviposição.
\end{abstract}

Palavras- chave: Rosaceae, mariposa-oriental, controle, terpenóides

ABSTRACT: Insecticidal potential of vegetable oil extracts on Grapholita molesta (Busck) (Lepidoptera: Tortricidae). As a result of the study on the chemical interactions between insects and herbivores and the advancement of phytochemical research studies, a variety of natural products with great insecticidal potential are described in the literature. The Grapholita molesta (Busck) (Lepidoptera: Tortricidae), commonly known as Oriental Fruit Moth, is a major peach pest damaging shoots and fruits. The purpose of this study was to assess the mortality, attractiveness and deterrence of vegetable oils in G. molesta. Insect eggs and pupae artificially created were immersed in the essential oils of Elionurus muticus (Spreng.) Kuntze and/or Cymbopogon winterianus Jowitt ex Bor at the concentrations of $0.25 \%, 0.5 \%, 1 \%$ and $0.5 \%, 1 \%$, $5 \%$, respectively. The attractiveness tests were performed in a Y-Tube olfactometer. Oviposition deterrence was tested in no-choice and dual-choice cages. Oil of $E$. muticus applied in eggs of G. molesta at three concentrations caused mortality above $30 \%$, which differs significantly from controls ( $p \leq 0.05)$. Mortality caused by citronella oil was approximately $70 \%$, significantly higher than control $(p \leq 0.01)$. The application of citronella oil at $1 \%$ in pupae caused mortality of $99.8 \%$. There was no attractiveness of adults in the olfactometer test for any of the oils. Citronella oil presented oviposition deterrence, as the mean number of eggs in the substrate with the treatment $(0.33 \pm 0.33)$ is significantly lower than in the control $(7.3 \pm 0.88)(p \leq 0.01)$.

Keywords: Rosaceae, oriental fruit moth, control, terpenoids.

\section{INTRODUÇÃO}

O cultivo de frutíferas temperadas no Brasil

tem sido realizado especialmente nos estados do sul do Brasil, como o Rio Grande do Sul, cujos cultivos de Rosaceae como a maçã (Pyrus malus L.) e o 
pêssego (Prunus persica (L.) Batsch) põem o estado em destaque na produção desses frutos (IBGE, 2005; João et al., 2001). Como fator limitante destas culturas destaca-se a Grapholita molesta (Busck) (Lepidoptera: Tortricidae), conhecida como "brocadas-ponteiras", em sua fase larval, ou, "mariposaoriental", na fase adulta, devido a sua origem asiática, sendo uma das mais importantes pragas mundiais de rosáceas. No início do século $X X$, já se destacava com impacto econômico negativo no Japão e, a partir de 1913, marcava presença no continente americano chegando ao Brasil em 1980 (Gonzalez, 1980). O dano é causado pelas larvas que após a eclosão migram para as brotações próximas penetrando-as, causando a destruição do meristema apical, a murcha, e apodrecimento dos ponteiros (Gonzalez, 2003).

Quando o ataque ocorre no pedúnculo este é penetrado pela larva formando túneis em direção ao fruto, atravessando o mesocarpo, e se alojando na região do caroço. O desenvolvimento das larvas ocorre no interior do fruto. A larva de $G$. molesta apresenta uma estrutura quitinosa no último segmento abdominal, denominado "pente anal", sendo diagnóstica para a sua distinção em relação a outras espécies em estado larval que utilizam o mesmo hospedeiro (Gonzalez, 1986; Nuñes \& Paullier, 1995).

Ao término da fase larval, cuja duração oscila entre oito a doze dias, a larva faz um orifício no fruto ou no ramo, e desce ao solo, através de uma linha de seda produzida por ela. No fruto, esse dano mecânico pode abrir janela para a atuação de patógenos, como o fungo Monilinia fructicola (Wint) (Botton et al., 2001), causando seu apodrecimento prematuro.

Para o manejo dessa praga têm-se adotado a utilização de inseticidas sintéticos fosforados e piretroides (Santa Cecília \& De Souza, 1985; Salles, 1998). Entretanto, atualmente intensificaram-se as pressões dos órgãos regulamentadores de saúde fixando novos conceitos de qualidade da produção e de segurança aos trabalhadores mediante a demanda e exigências dos centros consumidores por produtos de melhor qualidade. Diante dessa problemática, substâncias inseticidas de origem vegetal têm sido vistas como alternativas aos inseticidas sintéticos devido a sua biodegradabilidade e por constituírem menor risco a saúde humana (Murray \& Pinkham, 2002).

Óleos essenciais, em cuja constituição predominam substâncias voláteis, lipofílicas e de forte odor (Simões et al., 2007), são subprodutos do metabolismo secundário de diversas espécies de plantas, e, por não participar do metabolismo principal do vegetal, acabam por ser eliminados ou armazenados nas estruturas das plantas, tais como: raízes, caule e folhas.

O Rio Grande Sul apresenta vasta diversidade de espécies campestres naturais, sendo registradas aproximadamente 500 espécies de poáceas (LonghiWagner, 2003), das quais muitas são conhecidas e exploradas pela indústria de cosméticos e perfumaria. Destacam-se nesse cenário a citronela (Cymbopogon winterianus Jowitt ex Bor), Poaceae, e o capim-limão, Elionurus muticus (Sprengel) Kuntze, Poaceae, por apresentarem grande potencial fitoquímico.

Estas plantas têm em comum a presença do citral, componente majoritário do óleo essencial, conferindo ação antibacteriana e antifúngica (Negrelle \& Gomes, 2007), bem como de repelência a artrópodes vetores de doenças como Aedes aegypti (L.) (Diptera: Culicidae) (Tawatsin et al., 2001) e inibidor de oviposição em moscas-da-cebola Delia antiqua Meigen (Diptera: Anthomyiidae) (Cowles , 1990). No entanto, na literatura, os trabalhos referem-se principalmente a repelência utilizando óleo de citronela, sendo escassos os estudos mais detalhados de outros parâmetros em insetos. Testando diferentes concentrações do óleo de citronela sobre larvas de Spodoptera frugiperda (Smith) (Lepidoptera: Noctuidae) Labinas \& Crocomo (2002) encontraram mortalidade maior que $80 \%$ na concentração de $1 \%$.

Diante dos resultados satisfatórios desses óleos essenciais no controle de outros insetos, o presente trabalho teve como objetivo verificar a mortalidade, atratividade e deterrência provocada por óleos destes vegetais sobre a $G$. molesta.

\section{MATERIAL E MÉTODO}

\section{Criação de Grapholita molesta}

A criação foi mantida em câmara com condições controladas $\left(25 \pm 10^{\circ} \mathrm{C} ; 60 \pm 5 \%\right.$ U.R e fotofase de 16h), no Laboratório de Biologia, Ecologia e Controle Biológico de Insetos (BIOECOLAB), no Departamento de Fitossanidade, da Faculdade de Agronomia, UFRGS.

Pupas de machos e fêmeas de G. molesta foram armazenadas em "gaiolas" confeccionadas a partir de garrafas de plástico de $2 \mathrm{~L}$, cuja base foi retirada, mantendo-se um tecido do tipo voile no fundo, permitindo a renovação de ar e o manuseio. Após a emergência, no gargalo da garrafa, foi oferecido um algodão embebido com a dieta para adultos $\left(150 \mathrm{~g}\right.$ de mel para $1 \mathrm{~L}$ de água e Nipagin ${ }^{\circledR}$ (metilparabeno) a $0,15 \%$, segundo protocolo de criação do laboratório). Os adultos permaneceram na gaiola por dez a vinte dias, período em que as fêmeas realizaram a oviposição nas paredes desta. Posteriormente a retirada dos adultos das gaiolas, estas foram lavadas com água e hipoclorito de sódio a $15 \%$ e cortada em tiras, contendo ovos. As tiras

Rev. Bras. PI. Med., Campinas, v.16, n.2, p.182-188, 2014. 
foram depositadas em bandejas com dieta artificial para as larvas (Ivaldi-Sender, 1974). Entre cinco e sete dias depois dessa transferência, as bandejas foram envolvidas por tecido tipo gaze hidrófila, onde as larvas penetravam para empupar. As pupas foram coletadas em dez dias, antes da emergência de adultos e realocadas em gaiolas, reiniciando o ciclo de criação.

\section{Obtenção dos óleos}

Populações de capim-limão foram coletadas na região de São Borja, RS. Como não há descrição específica do $E$. muticus utilizado no experimento, a coleta realizada na mesma região confere segurança de que seja uma população da mesma espécie. A extração do óleo foi realizada no Laboratório de Horticultura e Silvicultura, da Faculdade de Agronomia da UFRGS, através do método de Hidrodestilação em aparelho tipo Clevenger. Após três horas de extração, obteve-se o rendimento de $2,5 \mathrm{~mL}$ de óleo essencial.

O óleo de Citronela foi obtido através da empresa comercial Raizando: óleos essenciais, de Minas Gerais, Brasil.

\section{Bioensaios}

Os bioensaios foram realizados com as fases de ovo, pupa e adulto, pois são estas que ficam expostas, em campo, à ação de produtos inseticidas, tendo em vista que a larva dificilmente recebe o contato destes produtos por estar protegida no interior do broto ou fruto.

\section{Mortalidade de ovos}

Para a aplicação do óleo de capim-limão em ovos de G. molesta, foram adotadas três concentrações de acordo com a disponibilidade de óleo: 0,25\% (0,2mL); 0,5\% $(0,4 \mathrm{~mL})$ e $1 \%(0,8 \mathrm{~mL})$, diluídas em $80 \mathrm{~mL}$ de veículo composto por $90 \%$ de água destilada $(72 \mathrm{~mL})$ e $10 \%$ de álcool etílico $(8 \mathrm{~mL})$. No controle 1 , as aplicações foram realizadas apenas com o veículo de diluição (água e álcool etílico), o controle 2 constou somente de água para averiguar se o álcool etílico exerceu algum efeito nos ovos. Das tiras retiradas das paredes das gaiolas foi feita a seleção de 20 ovos viáveis/ tira. A viabilidade foi determinada pela observação do encéfalo do embrião no interior dos ovos. Os demais ovos foram descartados com auxílio de agulha histológica. Utilizando-se uma pinça, cinco tiras contendo 20 ovos maduros cada uma, foram submetidas à imersão por 30 segundos, em cada uma das soluções com as concentrações do capimlimão. Transcorridas as aplicações, as tiras foram armazenadas em bandejas com dieta, na mesma câmara de criação, durante 10 dias. Após esse período foram feitas as contagens dos ovos que não eclodiram, caracterizando a morte destes.

Para o óleo de citronela a diluição foi realizada em $500 \mathrm{~mL}$ de veículo composto por $90 \%$ de água destilada $(450 \mathrm{~mL})$ e $10 \%$ de álcool etílico $(50 \mathrm{~mL})$. As concentrações adotadas foram adaptadas de Labinas \& Crocomo (2002) e Novelino et al. (2007), sendo $0,5 \%(2,5 \mathrm{~mL}), 1 \%(5 \mathrm{~mL})$ e $5 \%$ (25mL), além das aplicações com o veículo de diluição (controle). A técnica de aplicação do óleo de citronela sobre os ovos foi a mesma descrita para o capim-limão.

\section{Mortalidade de pupas}

O teste foi realizado comparando óleo de citronela com óleo mineral, pois não havia quantidade suficiente de óleo de capim-limão.

O óleo de citronela foi utilizado nas concentrações de $0,5 \%$ e $1 \%$. O óleo mineral Naturol (Farmax®), assim como anteriormente descrito para os óleos vegetais, foi diluído em $80 \mathrm{~mL}$ de veículo composto por $90 \%$ de água destilada $(72 \mathrm{~mL})$ e $10 \%$ de álcool etílico $(8 \mathrm{~mL})$, nas concentrações de $1 \%$ e $0,5 \%$. Foram feitas seis repetições em cada uma das concentrações utilizadas, compreendendo vinte pupas/repetição. As pupas foram envoltas em tecido tipo voile, e imersas pelo período de 20 segundos na solução. Após retirar da solução, esperou-se as pupas secarem e, em seguida, armazenou-se na câmara de criação. O mesmo procedimento foi realizado com as testemunhas, nas quais as pupas foram tratadas com água e álcool etílico. Após o período de dez dias realizou-se a contagem das pupas não emergidas, sendo estas consideradas mortas pelos tratamentos.

\section{Atratividade a adultos}

Visando verificar a atratividade dos dois óleos, utilizou-se um olfatômetro de vidro, em "y", de dupla escolha, onde os braços menores mediam $14 \mathrm{~cm}$ e o braço maior, $20 \mathrm{~cm}$. Abaixo desse dispositivo, colocou-se um papel milimétrico para controle da possível movimentação dos espécimes no interior do tubo. Nos braços do olfatômetro, colocou-se papel de filtro, de $9 \mathrm{~cm}^{2}$. Na extremidade aberta de um dos braços foi adicionado $10 \mu \mathrm{l}$ do veículo de diluição ( $90 \%$ água destilada e $10 \%$ de álcool etílico) e, no outro, $10 \mu \mathrm{l}$ de óleo essencial de citronela ou de capim-limão, nas concentrações de $1 \%$ e $0,5 \%$, respectivamente. Foram testados 18 machos e 18 fêmeas para cada tratamento. Individualmente, cada mariposa, de três a cinco dias de idade, foi adicionada ao sistema na posição inicial do olfatômetro. No primeiro momento, aguardouse o período de 10 minutos para aclimatação do espécime e esboço de movimentação. Não ocorrendo tal reação este indivíduo foi descartado, sendo substituído por outro. Havendo reação 
foram acrescidos cinco minutos para observação. Registrou-se o tempo que o inseto levou para alcançar a forquilha do olfatômetro, além do tempo gasto pelo inseto para percorrer pelo menos $4 \mathrm{~cm}$ no braço do olfatômetro contendo o tratamento com o óleo. A cada duas repetições, o sistema foi invertido $\left(180^{\circ}\right)$ a fim de anular possíveis pontos de referência externa que pudessem exercer influência nos indivíduos testados. O experimento foi realizado com 18 repetições para cada sexo e, após a sexta e décima segunda repetição, o olfatômetro foi desmontado, lavado e seco em estufa. Definiuse como resposta positiva ao tratamento, a permanência do espécime no interior do olfatômetro, com movimentação. A observação de tentativas de fugas do sistema caracterizou resposta negativa ao tratamento.

\section{Deterrência à oviposição}

O teste foi realizado somente com o óleo de citronela. No experimento sem escolha, as paredes internas de gaiolas confeccionadas com garrafas de plástico de $600 \mathrm{~mL}$ receberam, com auxílio de um pincel, a solução de óleo de citronela a $1 \%$. No experimento com dupla escolha, a garrafa foi cortada ao meio, longitudinalmente e parte desta recebeu o óleo e a outra parte, somente o veículo de diluição. Em seguida as partes foram unidas com fita adesiva. A gaiola controle recebeu somente o veículo de diluição. Com a gaiola seca, dez casais com 10 dias de idade foram colocados em cada garrafa onde permaneceram por $5 \mathrm{~h}$, no horário de pico da atividade reprodutiva (das 17 às 22h) (Herter, 1986). Foram realizadas três repetições por tratamento.

Após este período, os casais foram retirados da gaiola e os ovos colocados nas paredes das mesmas foram contabilizados.

\section{Análise dos dados}

Todos os experimentos foram realizados no delineamento experimental completamente casualizado. As porcentagens de redução da população (mortalidade) foram ajustadas através do software Ldp Line ${ }^{\circledR}$ (Finney, 1971) segundo fórmula proposta por Henderson \& Tilton (1955):

$$
E(\%)=100\left(1-B^{*}{ }^{*} U v / B r^{*} U n\right)
$$

onde $E(\%)$ é a eficiência dos tratamentos; Bn é o número de indivíduos das parcelas após o tratamento; $\mathrm{Br}$ é número de indivíduos das parcelas antes do tratamento; Un é número de indivíduos das testemunhas após o tratamento e Uv é a testemunha antes do tratamento.

Os dados foram testados quanto à normalidade pelo teste Lilliefors, que permite avaliar amostras com menos de dez unidades. A variação entre a média de insetos mortos foi submetida à análise de variância, comparando-se as médias pelo teste de Tukey através do software Bioestat 5.0 (Ayres et al., 2006).

\section{RESULTADOS}

\section{Mortalidade de ovos}

As aplicações dos dois óleos essenciais em ovos de G. molesta mostraram-se eficazes em todas as concentrações, sendo verificadas mortalidades significativamente maiores que as ocorridas nos dois controles (Tabela 1). As aplicações de óleo de citronela nas concentrações de $5 \%, 1 \%$ e $0,5 \%$, mostraram eficácia, apresentando mortalidades de $81 \%, 74 \%$ e $62 \%$, respectivamente. Nas concentrações de 0,5 e $1 \%$, o óleo de citronela teve maior efeito comparado com as mesmas concentrações de óleo de capim-limão (Tabela 1).

\section{Mortalidade de pupas}

A mortalidade de pupas (Tabela 2) não diferiu entre os óleos testados na diluição de $1 \%$. $\mathrm{Na}$ diluição de $0,5 \%$ a mortalidade foi significativamente maior para aquelas expostas ao óleo de citronela. Ambas apresentaram número médio de adultos emergidos menor do que as expostas ao controle com água e álcool etílico.

TABELA 1. Número médio (+EP) de ovos de Grapholita molesta mortos e mortalidade corrigida (\%) após tratamento com os óleos essenciais de citronela e de capim-limão, em várias concentrações.

\begin{tabular}{lllllll}
\hline $\begin{array}{c}\text { Concentração do } \\
\text { óleo (\%) }\end{array}$ & Controle 1 & Controle 2 & $\begin{array}{c}\text { Óleo de } \\
\text { Citronela }\end{array}$ & $\begin{array}{c}\text { Mortalidade } \\
(\%)\end{array}$ & $\begin{array}{c}\text { Óleo de } \\
\text { Capim- } \\
\text { Limão }\end{array}$ & $\begin{array}{c}\text { Mortalidade } \\
\text { (\%) }\end{array}$ \\
\hline 0,25 & $6,3 \pm 1,75 \mathrm{~b}$ & $4,8 \pm 1,64 \mathrm{bc}$ & - & - & $10,6 \pm 0,50 \mathrm{a}$ & 31 \\
0,5 & $6,3 \pm 1,75 \mathrm{c}$ & $4,8 \pm 1,64 \mathrm{~cd}$ & $14,8 \pm 0,37 \mathrm{a}^{*}$ & 62 & $11 \pm 0,54 \mathrm{~b}$ & 34 \\
1 & $6,3 \pm 1,75 \mathrm{c}$ & $4,8 \pm 1,64 \mathrm{~cd}$ & $16,4 \pm 0,24 \mathrm{a}^{* *}$ & 74 & $13,4 \pm 0,74 \mathrm{~b}$ & 52 \\
5 & $6,3 \pm 1,75 \mathrm{~b}$ & $4,8 \pm 1,64 \mathrm{bc}$ & $17,4 \pm 0,74 \mathrm{a}^{*}$ & 81 & - & - \\
\hline
\end{tabular}

Controle 1: água destilada+álcool etílico; Controle 2: água destilada; ${ }^{* *}$ * Médias seguidas de letras diferentes na linha diferem pelo teste de Tukey ( $p \leq 0,01$ e $p \leq 0,05$, respectivamente). 
TABELA 2. Número médio (+EP) de pupas de Grapholita molesta mortas e mortalidade corrigida (\%) após tratamento com o óleo essencial de citronela e mineral em duas concentrações.

\begin{tabular}{cccccccc}
\hline \multirow{2}{*}{$\begin{array}{c}\text { Concentração } \\
(\%)\end{array}$} & \multicolumn{2}{c}{ Óleo de citronela } & \multicolumn{2}{c}{ Óleo mineral } & \multicolumn{2}{c}{ Controle } \\
\cline { 2 - 8 } & $\begin{array}{c}\text { Pupas } \\
\text { mortas }\end{array}$ & $\begin{array}{c}\text { Mortalidade } \\
(\%)\end{array}$ & $\begin{array}{c}\text { Pupas } \\
\text { mortas }\end{array}$ & $\begin{array}{c}\text { Mortalidade } \\
\mathbf{( \% )}\end{array}$ & $\begin{array}{c}\text { Pupas } \\
\text { mortas }\end{array}$ & $\begin{array}{c}\text { Mortalidade } \\
(\%)\end{array}$ \\
\cline { 2 - 8 } 1 & $19,6 \pm 0,24 \mathrm{a}$ & 99,8 & $19,2 \pm 0,37 \mathrm{a}$ & 78,08 & $6,6 \pm 1,4 \mathrm{~b}^{*}$ & 45 \\
0,5 & $20,0 \pm 0,0 \mathrm{a}$ & 100 & $17,6 \pm 0,24 \mathrm{~b}$ & 61,82 & $6,6 \pm 1,4 \mathrm{c}^{*}$ & 45 \\
\hline
\end{tabular}

Controle: água destilada + álcool etílico. * Médias seguidas de letras diferentes na linha diferem pelo teste de Tukey $(p \leq 0,05)$

\section{Atratividade a adultos}

Não foi evidenciada atividade atrativa dos dois óleos adicionados no olfatômetro em "y", em nenhum dos 18 casais apresentados ao sistema. Foi observada (i) movimentação intensa e desorientada no interior do tubo;(ii) imobilidade e forte antenação ou (iii) fuga do sistema, indicando que os óleos utilizados não se apresentam como alternativa para atração em adultos de mariposa-oriental.

\section{Deterrência à oviposição}

No experimento sem escolha, o número médio de ovos na gaiola de oviposição tratada com óleo de citronela na concentração de $1 \%$, foi de $0,33 \pm 0,33$, sendo significativamente menor do que na testemunha $(7,3 \pm 0,88)(p \leq 0,01)$. No teste com dupla escolha, o número médio de ovos na parede da gaiola tratada com o referido óleo foi de $1,33 \pm 0,88$, diferente do registrado na parede da gaiola sem o tratamento, $2,69 \pm 1(p \leq 0,05)$

\section{DISCUSSÃO}

A mortalidade mais intensa apresentada pelo óleo de citronela em relação ao de capim-limão na concentração de $1 \%$ pode estar associada à presença mais marcante do elemento citral, em citronela. O detalhamento dos constituintes de um óleo essencial é extremamente importante devido à variação em sua composição. Sabe-se que o odor cítrico típico, apresentado por $E$. muticus e $C$. winterianus se deve a presença do elemento citral. Sua constituição resume-se ao sinergismo de dois subprodutos, o geranial e o neral. Segundo Füller (2008), a concentração média desses compostos em óleos provenientes de populações de capim-limão, oriundos de São Borja, obedece às proporções de $16 \%$ (geranial) e $9 \%$ (neral). Em citronela, os principais constituintes identificados no óleo foram os monoterpenos oxigenados, citronelal $(23,59 \%)$, geraniol $(18,81 \%)$ e citronelol $(11,74 \%)$ (Oliveira et al., 2011). Estas características explicariam o efeito diferencial dos óleos.

O efeito ovicida apresentado pelo óleo de citronela nas doses de 0,5 e $1 \%$ foi considerado satisfatório, tendo em vista a ineficiência de inseticidas clássicos utilizados, no controle destas pragas, como relataram Arioli et al. (2007) que, utilizando o inseticida sintético Imidan 500 PM a $2 \%$, não observaram mortalidade dos ovos de $G$. molesta. Embora a exposição aos tratamentos tenha sido menor (10s), a total ausência de mortalidade mostra a pouca eficácia desse inseticida sintético na mortalidade de ovos de G. molesta, contrastando com o potencial ovicida dos óleos das poáceas analisadas.

A utilização do álcool etílico adicionado na água destilada exerceu efeito co-solvente auxiliando na diluição e não exerceu efeitos significativos sobre os ovos.

A grande mortalidade de pupas obtida no tratamento com óleo de citronela está relacionada à interrupção metabólica da pupa causada pelo óleo, associada ao efeito tóxico da citronela. De acordo com Teixeira et al. (2005) a tenacidade dos óleos tende a ser mais intensa em tubulações (viscosidade mecânica), como nos canais traqueais das pupas. Desse modo, o contato do óleo com a pupa pode ter obstruído os espiráculos, ou até mesmo penetrado nos canais traqueais, cessando total ou parcialmente a respiração, levando ao comprometimento funcional e morte da pupa. A diferença de mortalidade encontrada nas concentrações de $0,5 \%$ do óleo de citronela e de $0,5 \%$ do óleo mineral sugere que o maior efeito obtido no primeiro tratamento foi devido à ação tóxica do princípio ativo da citronela. Por conseguinte, os óleos essenciais podem ter ação dupla na mortalidade da fase de pupa. Em criações artificiais a mortalidade de pupas pode chegar a $27 \%$ dependendo do substrato alimentar das larvas (Bisognin et al., 2012). Assim, a mortalidade apresentada pelo controle utilizado $(45 \%)$ pode indicar que este teve efeito sobre a viabilidade das pupas o que não ocorreu em relação aos ovos. A mortalidade causada por extratos alcoólicos de plantas é discutida em diferentes trabalhos, tais como o de Almeida et al. (2004) em grãos armazenados, entretanto, os autores não distinguem o efeito isolado do álcool etílico no resultado. 
Em relação aos resultados apresentados para atratividade de fêmeas, Altafini et al. (2009) verificaram adaptação satisfatória de G. molesta em olfatômetro em "y", onde os espécimes, expostos no tubo com extratos das plantas hospedeiras, se locomoveram em direção a fonte do extrato. No entanto, a movimentação comportamental dos indivíduos, utilizando os óleos de citronela e de capim-limão, não foi equânime ao estudo citado.

A diferença significativa no tratamento de dupla escolha, entre a região tratada e não na tratada, pode estar associada ao comportamento avaliativo de substrato pré-postura. A escolha do local de oviposição é tão importante quanto a quantidade ou a viabilidade dos ovos. Segundo Blaney \& Simmonds (1990), a escolha de um local se deve a presença de quimioreceptores tarsais que interagem com uma infinidade de aleloquímicos, interpretando possíveis localidades para a postura. Portanto, o efeito deterrente do óleo de citronela sobre a oviposição pode ser importante ferramenta para prevenir o estabelecimento de G. molesta. Cabe ressaltar a ocorrência de mortalidade dos adultos na gaiola tratada, podendo, desse modo, mascarar os resultados. Por outro lado, essa mortalidade pode indicar a ação do óleo sobre os adultos da mariposa, uma vez que há relatos de mortalidades em adultos de afídeos, tripes, mosca-branca e ácaros (Cloyd et al., 2009; Gorski \& Tomczak, 2010).

A partir dos resultados obtidos pode-se inferir que os óleos de citronela e de capim-limão têm potencial para o controle de G. molesta nas fases de ovo, pupa e adulto, as quais, em campo, são as que ficam mais expostas à ação dos produtos.

\section{AGRADECIMENTOS}

À Thanise Füller pelo apoio nas extrações dos óleos de capim-limão e à Raizando Óleos Essenciais, pelo patrocínio e fornecimento do óleo de citronela.

\section{REFERÊNCIA}

ALMEIDA, S.A. et al. Atividade inseticida de extratos vegetais sobre Callosobruchus maculatus (Fabr., 1775) (Coleoptera: Bruchidae) Revista Brasileira de Agrociência, v.10, n.1, p.67-70, 2004.

ALTAFINI, D.L.; SANT'ANA, J.; RADAELLI, L.R. Influência de fatores endógenos na percepção eletrofisiológica e comportamental de Grapholita molesta (Buck) (Lepidoptera: Tortricidae) a voláteis de plantas hospedeiras. Arquivos do Instituto Biológico, v.76, n.4, p. 651-48, 2009.

ARIOLI, C. J. et al. Técnica de criação de Grapholita molesta (Busck, 1916) (Lepidoptera: Tortricidae) em laboratório utilizando dieta artificial para produção de insetos visando estudos de comportamento e controle. Boletim de Pesquisa e Desenvolvimento, Embrapa Uva e Vinho, n.13, 2007.

AYRES, M. et al. BioEstat 4.0: aplicações estatísticas nas áreas da ciência biológica e médica. Sociedade Civil Mamirauá/CNPq, Belém, Brasil, 2006. 324p.

BLANEY, W.M.; SIMMONDS, M.S.J. A behavioral and electrophysiological study of the role of tarsal chemoreceptors in feeding by adults of Spodoptera littoralis, Heliothis virescens and Helicoverpa armigera. Journal of Insect Physiology, v.36, p.743-56, 1990.

BISOGNIN, M. et al. Burrknots as Food Source for Larval Development of Grapholita molesta (Lepidoptera: Tortricidae) on Apple Trees. Environmental Entomology, v.41, n.4, p. 849-54, 2012.

BOTTON, M.; ARIOLLI, C.J.; COLLETTA, V.D. Monitoramento da mariposa oriental Grapholita molesta (Busck, 1916) na cultura do pessegueiro. Comunicado Técnico Embrapa Uva e Vinho Bento Gonçalves, v.38, p.4, 2001.

CLOYD, R. A. et al. Effect of Commercially Available Plant-Derived Essential Oil Products on Arthropod Pests. Journal of Economic Entomology, v.102, n. 4, p.1567-79, 2009.

COWLES, R.S. Manipulating oviposition of the onion fly, Delia antiqua (Meigen) by stimulo deterrent diversion.1990. 330p. PhD thesis. Michigan State University, East Lansing.

FINNEY, D. J. Probit analysis. 3 ed. Cambridge University Press, Cambridge, London. 1971, 333p.

FÜLLER, T. N. Caracterização fenotípica, fitoquímica e molecular de Elionurus sp. HUMB. \& BOMPL ex WILD (Capim-limão), 2008. 66p. Dissertação (Mestrado) - Programa de Pós Graduação em Fitotecnia, Universidade Federal do Rio Grande do Sul, Porto Alegre.

GONZALEZ, R. H. La grafolita, una nueva polilla de la manzana en Curicó. Fruticola, v.1, n.2, p.7-11, 1980.

GONZALEZ, R. H. Fenologia de la polilla oriental del durazno. Aconex, n.12, p.5-12, 1986.

GONZALEZ, R. H. Las polillas de la fruta em Chile (Lepidoptera: Tortricidae; Pyralidae). Santiago: Facultad de Ciências Agronômicas de Chile, 2003. $188 \mathrm{p}$.

GORSKI, R.; TOMCZAK, M. Usefulness of natural essential oils in the control of foxglove aphid (Aulacorthum solani Kalt.) occurring on eggplant (Solanum melongena L.). Ecological Chemistry and Engineering S-Chemia I Inzynieria Ekologiczna S, v.17, n.3, p.345-49, 2010.

HENDERSON, C.F.; TILTON E.W. Tests with acaricides against the brow wheat mite, Journal of Economical Entomology, v.48, p.157-61, 1955.

HERTER, F.G. et al. Horário de Captura de Grapholita molesta em Pessegueiro. In: CONGRESSO BRASILEIRO DE ENTOMOLOGIA, 10., 1986, Rio de Janeiro. Resumos... Rio de Janeiro, SEB,1986. p.11.

IBGE. Pesquisa mensal de previsão e acompanhamento das safras agrícolas no ano civil: janeiro 2005. Levantamento Sistemático da Produção Agrícola, v.17, p.1-62, 2005. Disponível em <ftp://ftp.ibge.gov.br/ Producao_Agricola>. Acesso em: 8 nov. 2010.

IVALDI-SENDER, C. Techniques simples pour um élevage permanent de la tordeuse orientale, Grapholita molesta

Rev. Bras. PI. Med., Campinas, v.16, n.2, p.182-188, 2014. 
(Lepidoptera, Tortricidae) sur milieu artificiel. Annales

Zoologie Ecologie Animale, v.6 n.2, p.337-43, 1974.

JOÃO, P.L. et al. Levantamento da fruticultura comercial do Rio Grande do Sul. Porto Alegre, EMATER, (Série Realidade Rural), n.28, 2001. 80p.

LABINAS, A.M.; CROCOMO, W.B. Effect of java grass (Cymbopogon winterianus Jowitt) essential oil on fall armyworm Spodoptera frugiperda (J. E. Smith, 1797) (Lepidoptera, Noctuidae). Acta Scientiarum, v.24, n.5, p.1401-05, 2002.

LONGHI-WAGNER, H. M. Diversidade Florística dos campos sul-brasileiros: Poaceae. In: CONGRESSO NACIONAL DE BOTÃNICA, 54., 2003, Belém. Resumos. Sociedade Botânica do Brasil, 2003. p.117-20.

MURRAY, C.; PINKHAM, C. Towards a Decision Support Tool to Address Invasive Species in Garry Oak \& Associated Ecosystems in BC. Prepared by ESSA Technologies Ltd. Victoria, B.C. for the GOERT Invasive Species Steering Committee, Victoria, B.C., 2002. 96 p.

NEGRELLE, R.R.B.; GOMES, E.C. Cymbopogon citratus (DC) Stapf: chemical and pharmacological review. Revista Brasileira de Plantas Medicinais, v.9, n.1, p.80-92, 2007.

NOVELINO, A.M.S.; DAEMON, E.; SOARES, G.L.G. Avaliação da atividade repelente do timol, mentol, salicilato de metila e ácido salicilico sobre larvas de Boophilus microplus (Canestrini, 1887) (Acari:
Ixodidae). Arquivo Brasileiro de Medicina Veterinária e Zootecnia, v.59, p. 700-4, 2007.

NUÑES, S.; PAULLIER, J. Cydia molesta (BUSK). In: BEMTANCOURT, C. M.; SCATONI I.B (Ed.). Lepidopteros de importância econômica: reconocimiento biologia y daños de lãs plagas agrícolas y florestales. Uruguai: Agropecuaria Hemisfério Sur S.R.L., 1995. p.32-40.

OLIVEIRA, W.A. et al. Antifungal activity of Cymbopogon winterianus Jowitt ex bor against Candida albicans. Brazilian Journal of Microbiology, v.42, p433-41, 2011.

SALLES, L. A. B. Principais pragas e seu controle. In: MEDEIROS, C.A.; RASEIRA, M.C.B. (Eds.). A cultura do pessegueiro. Pelotas: Embrapa Clima Temperado, 1998. p.205-39.

SANTA CECÍLIA, L.V.C.; DE SOUZA, J.C. Pragas das frutíferas de clima temperado. Informe Agropecuário, v.11, n.125, p.43-56, 1985.

SIMÕES, C.M.O. et al. Farmacognosia: da planta ao medicamento. Florianópolis: Editora da UFSC, 2007. 1102p.

TAWATSIN, A. et al. Repellency of volatile oils from plants against three mosquito vectors. Journal of Vector Ecology, v.26, n.1, p.76-82, 2001.

TEIXEIRA, O.P.B. et al. Mecânica dos fluidos: algumas considerações sobre a viscosidade. In: XVI Simpósio Nacional de Ensino de Física, 16., 2005, Rio de Janeiro. Resumos... Rio de Janeiro: Sociedade Brasileira de Física, 2005. p.171. 